\title{
Progress update of the VGOS radio telescope at Metsähovi Geodetic Research Station
}

\section{Guifré Molera Calvés*†}

Finnish Geospatial Research Institute, National Land Survey of Finland.

University of Tasmania, School of Natural Sciences, Department of Maths and Phyisics.

E-mail: Guifre.MoleraCalveseutas.edu.au

\begin{abstract}
The three-year project of building a VGOS radio telescope system at the Metsähovi Geodetic Research Station, Finland, is progressing as planned. The installation site was ready for the telescope arrival with the foundation and the anchor ring for the antenna's steel pedestal already on site by the end of 2017. The manufacturing of the telescope was completed by MT Mechatronics $\mathrm{GmbH}$ and it was delivered on-site in June 2018. Telescope assembly and installation was done during the summer and finalized in October 2018. The assembly of the signal chain components also moved forward. The installation and integration of the signal chain is scheduled for the spring 2019, and it is expected to have first observational tests by the end of 2019. This paper presents the latest project status and describe the next steps for the Metsähovi VGOS station.
\end{abstract}

14th European VLBI Network Symposium \& Users Meeting (EVN 2018)

8-11 October 2018

Granada, Spain

* Speaker.

${ }^{\dagger}$ A footnote may follow. 


\section{Introduction}

The Metsähovi Geodetic Research Station is a key infrastructure of the Finnish Geospatial Research Institute (FGI), part of the National Land Survey of Finland (NLS). Metsähovi is located in the Southern part of Finland at a Latitude $60^{\circ} 13^{\prime} 2.9^{\prime \prime}$, Longitude of $24^{\circ} 23^{\prime} 43.1^{\prime \prime}$, and altitude of $95 \mathrm{~m}$. Metsähovi is a Global Geodetic Observing System (GGOS) core site, i.e., member of global network of geodetic stations which are used to maintain global terrestrial and celestial reference frames, compute precise orbits of satellites, and for geophysical studies.

Metsähovi is one of the few geodetic stations that has all major geodetic observing instrumentation's collocated. These include satellite laser ranging (SLR), very long baseline interferometry (VLBI), global navigation satellite system (GNSS), superconducting and absolute gravimeters, and the Doppler Orbitography and Radio positioning Integrated by Satellite (DORIS) beacon. The station has contributed since 1978 to several global services of the International Association of Geodesy (IAG), and due to its long existence it helps to retain sustainability in the maintenance of global reference frames.

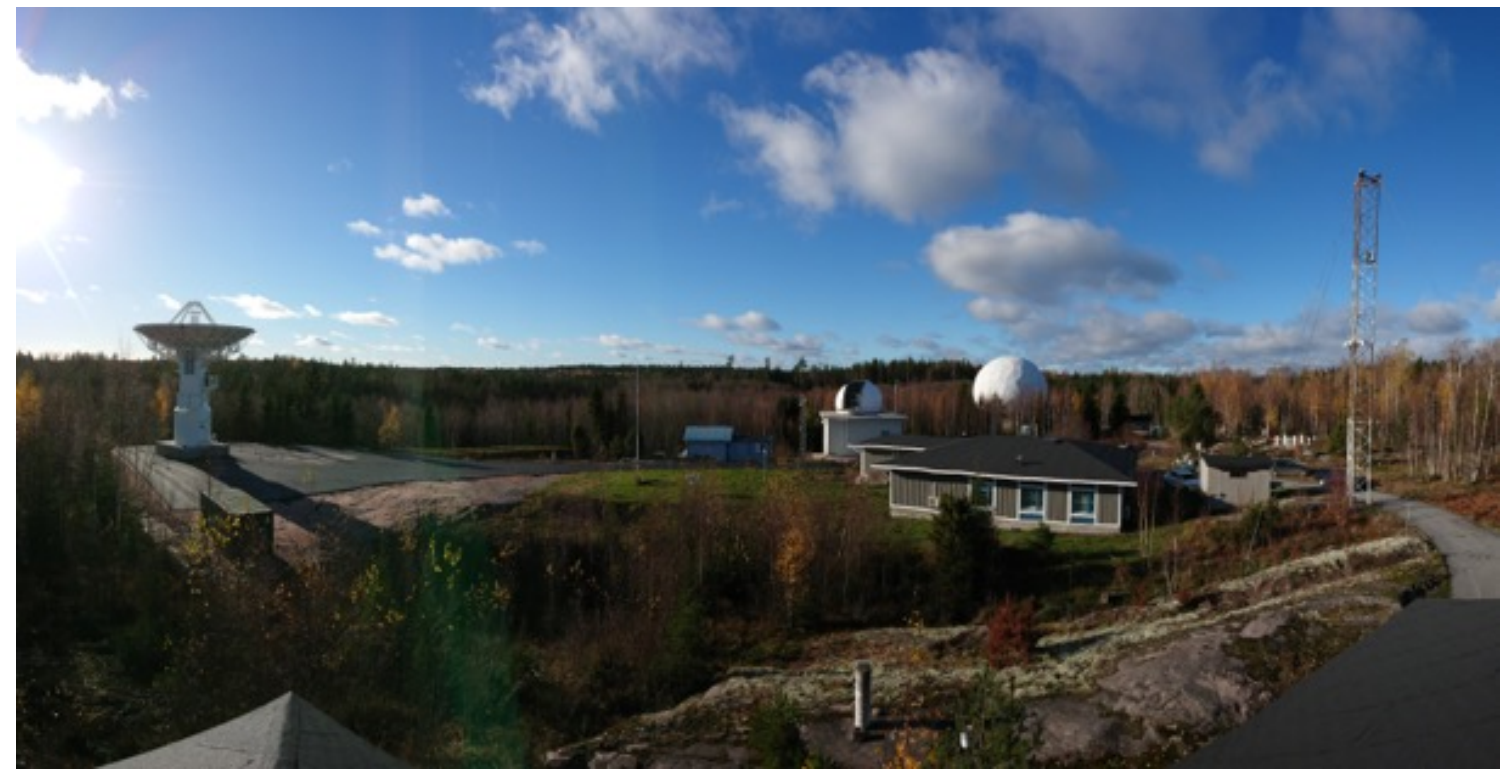

Figure 1: Overview of the Metsähovi Geodetic Research Station. The new VGOS radio telescope is on the left side, the main building with all the computer facilities (middle), the small radome behind the main building belongs to the SLR, and finally the big radome is part of the Aalto Metsähovi Radio Observatory. Photo: Markku Poutanen

\section{Background}

FGI has been actively participating in the International VLBI Service for Geodesy and Astrometry (IVS) global observations since early 2000's. During that time FGI did not have yet own radio telescope, and instead rented the observational time to the Aalto Metsähovi Radio Observatory (MRO). MRO has an operational 14-metre radio telescope since 1979, which is primarily 
dedicated to radio astronomy purposes. During this past decade FGI took part in the IVS program with an average of four to six observing campaigns per year.

FGI began the process of searching for funding in 2009 for the complete renewal of the Metsähovi Geodetic Research Station. The first batch of money allowed the construction of the new SLR station (which concluded in 2015), the acquisition of an absolute gravimetre and superconducting gravimeter, as well as, a major upgrade of the infrastructure of the station itself. In 2015 the Ministry of Forestry and Agriculture provided a special funding for the construction of a new VGOS radio telescope [1].

The official start of the project was in January 2016 with the tendering process to select the manufacturer of the antenna. The process lasted approximately 11 months and finally MT Mechatronics GmbH (MTM) was selected as the main constructor of the new radio telescope. It is the ninth VGOS-type antenna designed and built by the manufacturer, after three in Spain, two in Sweden, two in Norway, and one in South Africa.

\section{Construction}

The site for the construction of the new antenna is within 100 metres from the other infrastructures at the Metsähovi Geodetic Research Station, and $500 \mathrm{~m}$ from the 14-m MRO antenna. (see Figure 1). All the Metsähovi area is laying over bedrock, so it was easy to select a good spot for the antenna. The preparation work for the on-site area began in summer 2017 and were complete by the end of 2017. Figure 3 shows the status of the construction site in summer 2017, when all the vegetation and dirt had been removed (left image), and in December 2017 when the final foundation platform with the proper anchor ring that fixes the radio telescope with the bedrock was complete (right image).
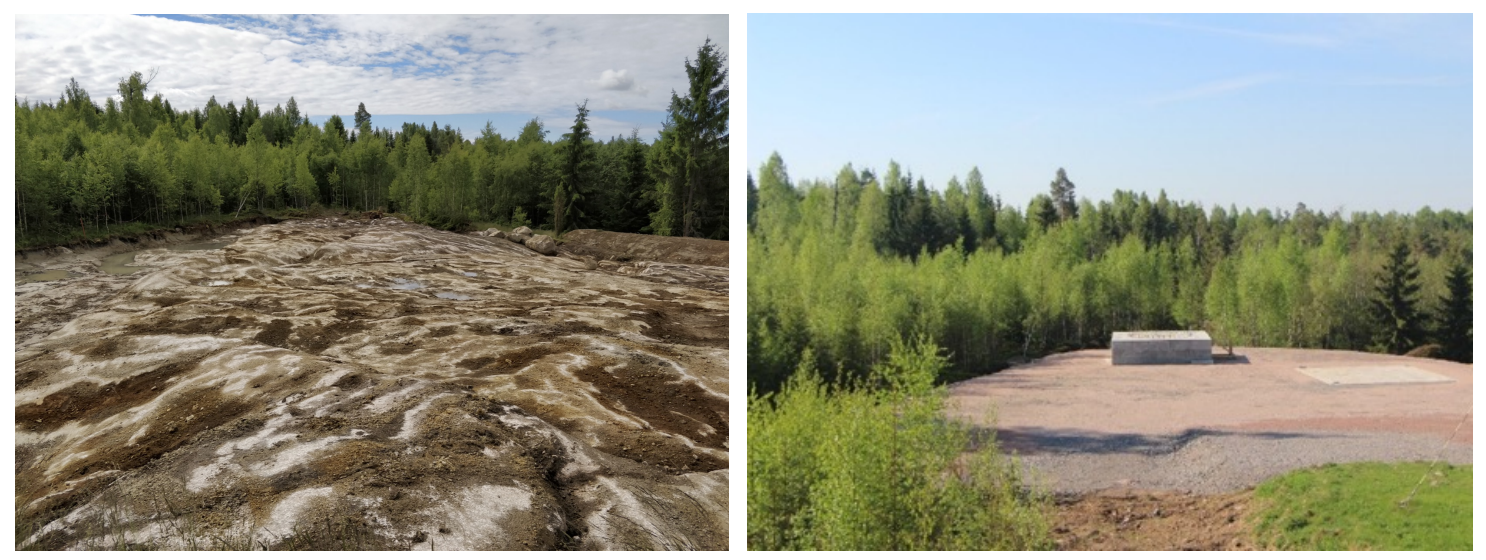

Figure 2: Left- site of the antenna construction after removal of the dirt and vegetation, summer 2017. Right- site ready for the installation of the new antenna, summer 2018. Photo: Guifré Molera Calvés.

The on-site preparations work included also the installation of the electrical (main and UPS) power lines to the radio telescope and the links for the communication signals. The radio telescope is connected to the main building thanks to four optic fibres cable and 1 coaxial cable. The optic fibres are of two different types: exclusively monomodes (2x12 SMF) and mixed (2x6SMF + 
$2 \mathrm{xMMF}$ ). Three of the fibres terminate at the basement of the tower, while the other one is directly routed to the elevation cabin.

All the mechanical components of the antenna and the servo equipment arrived at Metsähovi at the ends of June 2018. The installation of the new radio telescope by Chinese crew immediately after that. The construction of the was a success, and no major issues or delays were encountered during the process. The first photogrametric map of the main reflector surface showed a performance better than the requested in the tendering process.
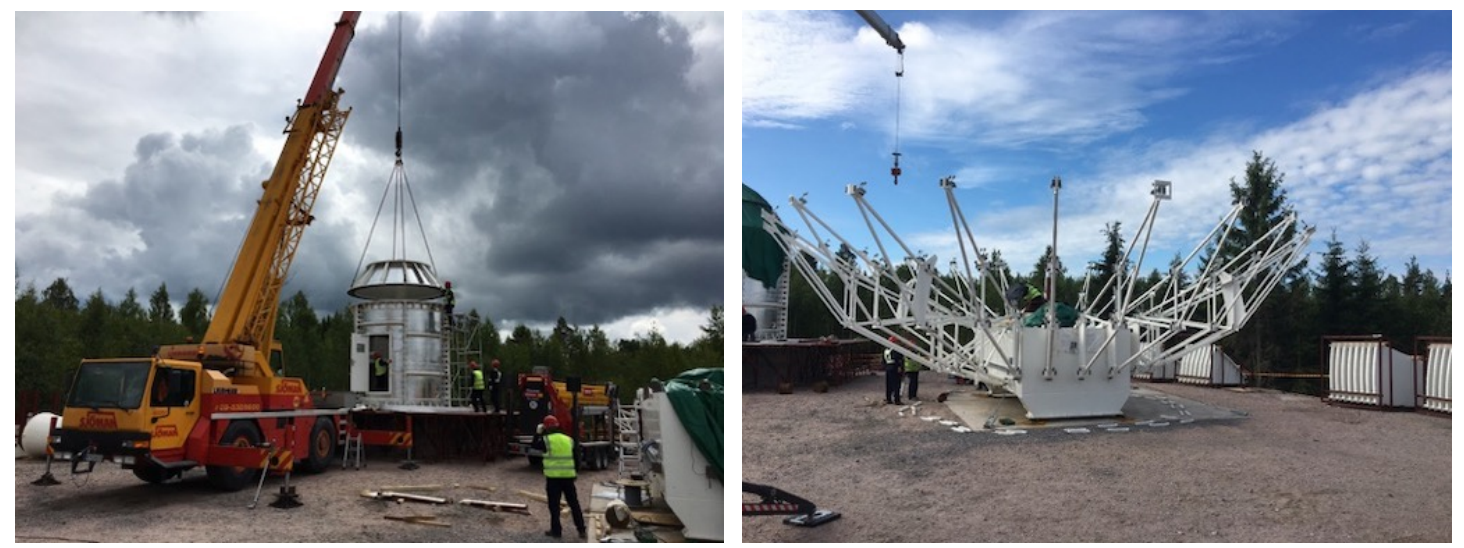

i) ii)

iii) iv)
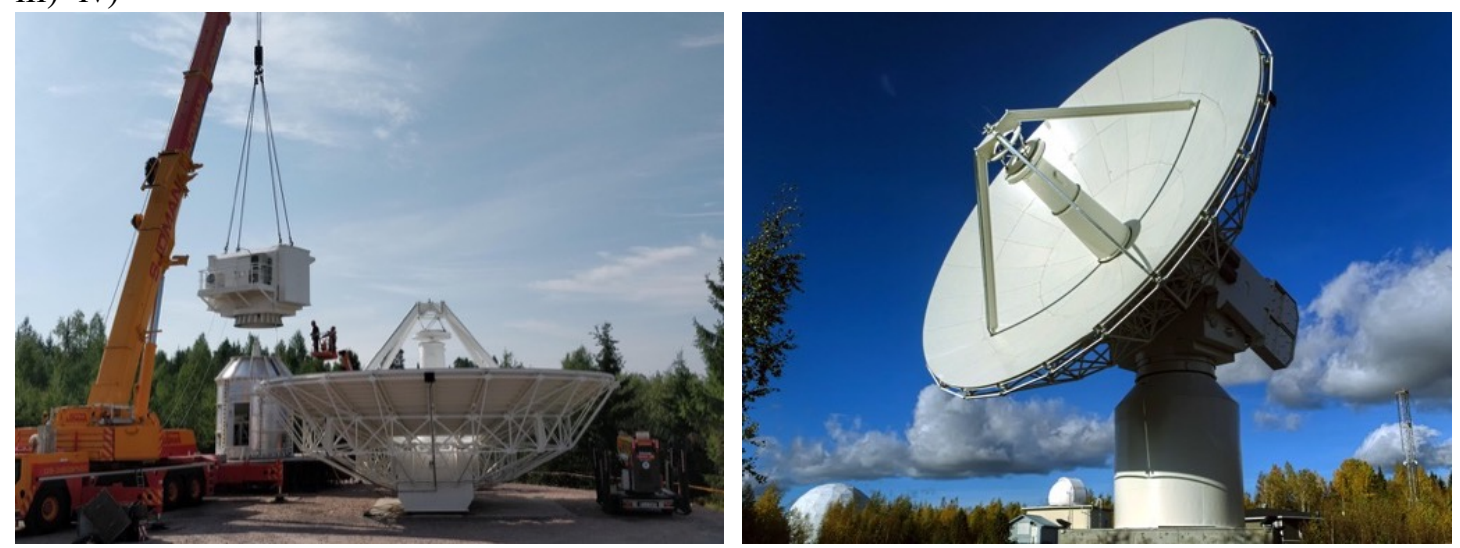

Figure 3: i) Week 1: Assembly of the antenna steel pedestal and the upper cone. ii) Week 2: Installation of the main reflector structure and assembly of the surface panels. iii) Week 4: The big lifts which included the final assembly of the azimuth, the ballast arms and the main reflector. iv) Week 10: Status of the radio telescope after the completion of the work in September 2018. Photos: Guifré Molera Calvés.

The Site Acceptance Test (SAT) of the radio telescope is expected to occur in December 2018. In case of a satisfactory scenario it is expected to start soon with the installation of the signal chain and the first tests.

\section{Signal chain}

An agreement with the Instituto Geografico Nacional (IGN) Center of Astronomy of Yebes 
(CAY) was reached in order to manufacturer the future front-end for the VGOS antenna at Metsähovi. The front-end will consist on a broadband receiver with a frequency of operations between 2.4 and $14.1 \mathrm{GHz}$ with a quad-ridge feed horn (QRFH). The signal will acquire Radio Frequency (RF) signals in both horizontal and vertical linear polarisations. The schematics of the broadband receiver from IGN is shown in Figure 4.
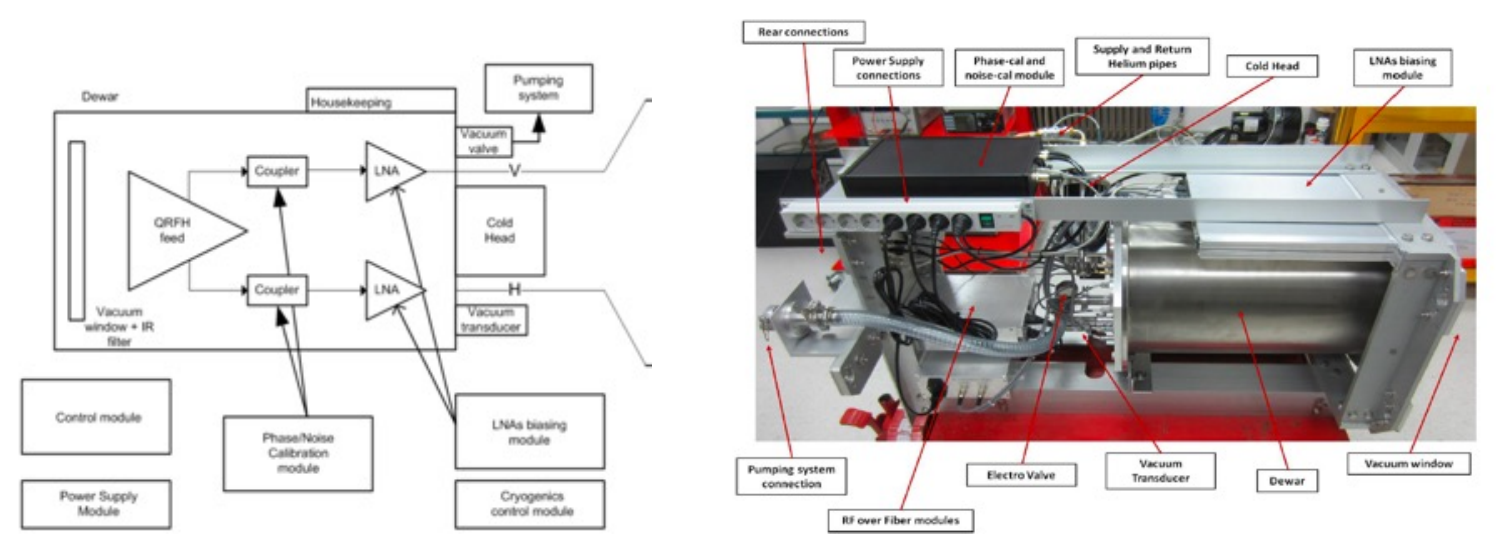

Figure 4: i) Schematics of the new broadband receiver for the VGOS radio telescope. The receive is being assembled by the Centro Astonomico de Yebes (IGN-CAY). ii) Current image of the existing broadband receiver from the Yebes VGOS antenna. Images: IGN.

The RF signal captured by the receiver is sent via optic fibre, with the Radio Frequency over Fiber technique (RFoF), to the main building for the digital sampling. However, prior, the signal is filtered and divided into lower and higher frequency bands, in order to avoid saturation in the fibre channels. We selected a low frequency channel up to $4 \mathrm{GHz}$ and a high frequency one from 4 to $15 \mathrm{GHz}$. These frequency bands were chosen in order to comply with the requisites of the digital baseband converter (dBBC3).

The RFoF receiver unit at the main building will convert the optical signal back to RF with $4 \mathrm{RF}$ signals (two polarisations and two frequency bands). The higher frequency bands will be filtered again, amplified and split in order to have three overlapping $4 \mathrm{GHz}$ bands for the dBBC3. The final bandwidth and frequency limits of these bands have not yet been completely decided a day of today.

As mentioned, Metsähovi will have a full compatible VGOS-type digital BBC (dBBC3) that will filter, digitize, sample and finally packetize the data into VLBI compatible format (VDIF format). A full compatible $\mathrm{dBBC} 3$ means that it will accept 2 polarisations and four times $4 \mathrm{GHz}$ input frequency bands. Depending on the observing mode the output of the $\mathrm{dBBC} 3$ can be from 1 to $324 \mathrm{Gbps}$ of stream of data output, being 8 the most common for the time being. In order to cope with high data flow we have selected a Mellanox SN2100 switch which will be the interface between the dBBC3, the Data Acquisition System (DAS) and the Internet.

We decided to use a FlexBuff system as our main recorder. It means that all data recorded must be transferred electronically to the correlator, and therefore, there is no possibility to ship disks via courier service. Currently we are not connected to the Finnish University and Research Network (FUNET) and its fast network connectivity, but, this will change in 2019 when the telescope should 


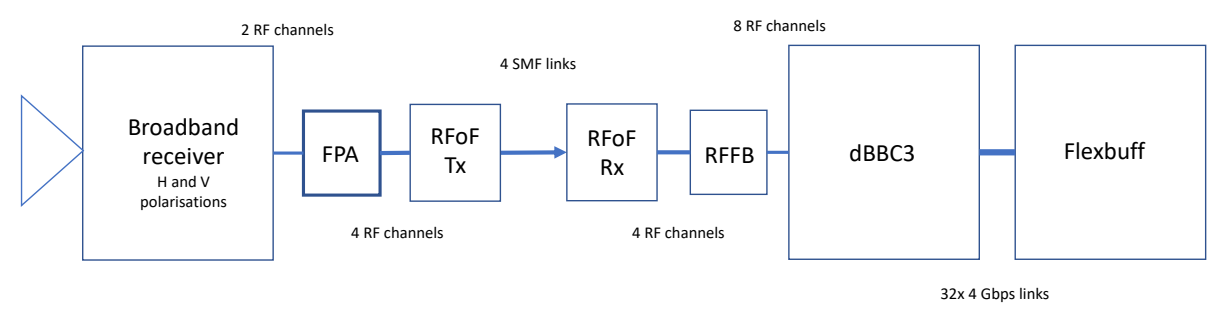

Figure 5: Complete picture of the signal chain at the VGOS Metsähovi Geodetic Research Station: Broadband receiver, RFoF modules, dBBC3 and FlexBuff.

be operational. The FlexBuff consists on two chassis one with the processing unit and the other with all the disks. There is capacity to allocate 44 disks. It is expected to have a 440 TB or larger when the telescope starts its operations.

\section{Time and Frequency distribution at Metsähovi}

The Metsähovi Geodetic Research Station will not have their own Hydrogen-maser for the VLBI observations. As it is known, the VLBI stations have relied on the precision of these clocks to provide timestamp information on the data and also a stable reference frequency to the system. Instead FGI made some effort on developing together with the Finnish Metrology Institute (MIKES/VTT) distribution of the official Finnish time via optic fibres to the station.

The system takes benefit of the White Rabbit technologies developed by CERN and commerciaized by SevenSols ${ }^{1}$. The White Rabbit Switch (WRS) allows the distribution from a so-called master node of the $1 \mathrm{pps}$ and 10 or $5 \mathrm{MHz}$ reference signal to any remote node connected to the switch via optic fibre $[2,3]$. The performance of these units can cope with the most demanding applications worldwide, among them VLBI observations. MIKES/VTT is located $35 \mathrm{~km}$ from Metsähovi and a pair of dark fibres connects the Institute with Aalto MRO. The connectivity and distribution plan of the clock signal is shown in the Figure 5.

Constant monitoring of the $1 \mathrm{pps}$ and $10 \mathrm{MHz}$ signal at Metsähovi has been conducted since summer 2017. Long stability analysis showed that the performance of the WRS with the addition of a Low Jitter board for improved clock stability on-board is only an order of magnitude worst than the performance of an Hydrogen-maser. However, this performance is sufficient to fulfill the VGOS requirements [4].

\section{Conclusions}

The planning, design and installation work of the new VGOS radio telescope at Metsähovi Geodetic Research Station has advanced according to the plan. The antenna will be completed by the ends of 2018 and first tests will be conducted during 2019. The installation of the broadband

\footnotetext{
${ }^{1}$ www.sevensols:com
} 


\section{Metsähovi (FGI)}

\section{White Rabbit Topology}

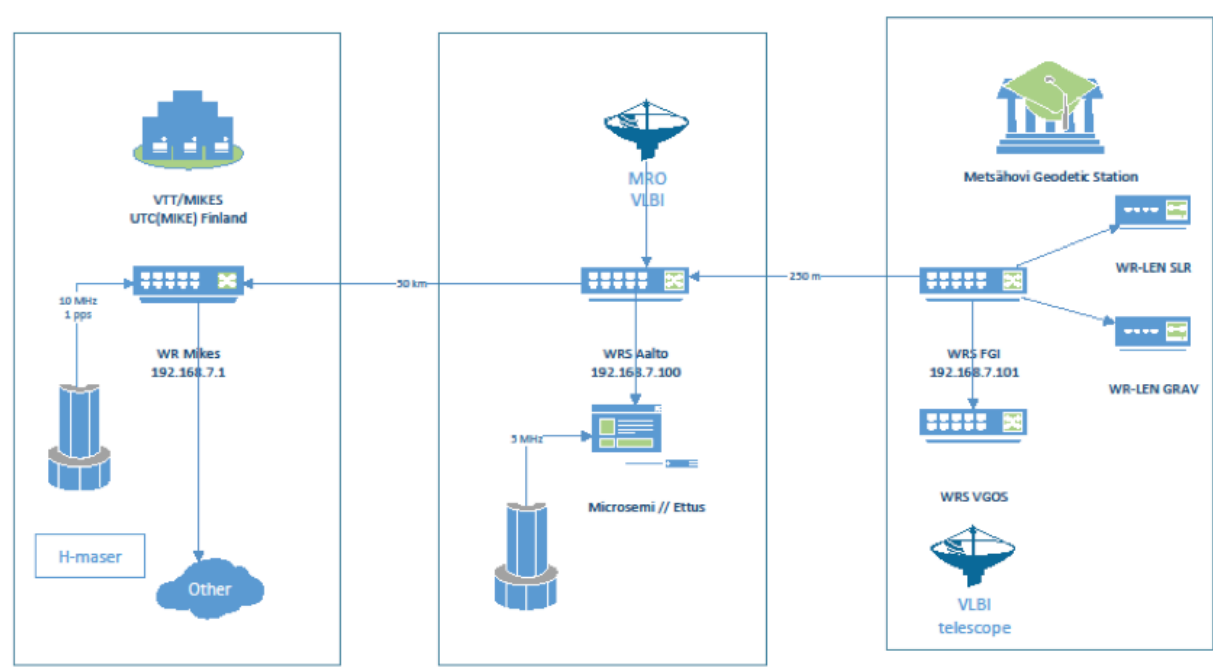

Figure 6: White Rabbit network topology to distribute the $1 \mathrm{pps}$ and the $10 \mathrm{MHz}$ reference signal from the Finnish Metrology Institute to the Metsähovi Geodetic Research Station. The distance between both institutes is $35 \mathrm{~km}$. More than a year of tests has demonstrated that this technology can cope with the needs of VGOS.

receiver provided by IGN is scheduled by spring 2019 and the antenna's first-light is expected during 2019.

It is expected that by 2020 the new VGOS radio telescope at Metsähovi will be fully functional to join the VGOS IVS observing campaigns.

\section{References}

[1] Petrov et al. Progress report of the IVS VLBI 2010 committee. Designs aspects of the VLBI 2010 system. NASA/TM-2009-214180

[2] M. Rizzi et al., "WRS Low Jitter Daughterboard", Open Hardware repository, https://www.ohwr.org/projects/wrslow-jitter

[3] Wallin, A.E. et al., Improved systematic and random errors for long-distance time-transfer using PTP White Rabbit, EFTF2018

[4] Molera Calvés, G. et al., Initial results from the MIKES-Metsähovi time and frequency link for the VGOS radio telescope, International VLBI Service General meeting, Svalbard 2018 